\title{
Smoothing Segmented Lung Boundary in Chest CT Images Using Scan Line Search
}

\author{
Yeny Yim $^{1}$ and Helen Hong ${ }^{2, \star}$ \\ ${ }^{1}$ School of Computer Science and Engineering, Seoul National Univ., \\ San 56-1, Shinlim-dong, Kwanakgu, Seoul 151-742, Korea \\ shine@cglab.snu.ac.kr \\ 2 Division of Multimedia Engineering, College of Information and Media, \\ Seoul Woman's Univ., 126 Gongreung-dong, Nowon-gu, Seoul 139-774, Korea \\ hlhong@swu.ac.kr
}

\begin{abstract}
To smooth lung boundary segmented by gray-level processing in chest CT images, we propose a new method using scan line search. Our method consists of three main steps. First, lung boundary is extracted by our automatic segmentation method. Second, segmented lung contour is smoothed in each axial CT slice. Scan line search is proposed to track the points on lung contour and find rapidly changing curvature without conventional contour tracking. 2D closing in axial CT slice is applied to reduce the number of rapidly changing curvature points. Finally, to provide consistent appearance between lung contours in neighboring axial slices, 2D closing in coronal CT slice is applied within pre-defined subvolume. Experimental results show that the smoothness of lung contour considerably increased after applying proposed method.
\end{abstract}

\section{Introduction}

Recently, high-resolution X-ray computed tomography (CT) images provide us a detailed abnormal area, and they are increasingly used for applications such as lung nodule detection, lung motion analysis. A critical first step in these applications is the automatic lung segmentation. To separate low density lung regions from the denser surrounding anatomy in chest CT images, most of methods use the difference in gray-level value of pixels $112 / 3$. In gray-level based method, radiodense pulmonary vessels near mediastinum and pleural nodules that contact with the surrounding anatomy, as shown in Fig. 1(a) and (b), are excluded from the segmentation result. This can bring about indentations on segmented lung regions. On the other hand, gray-level based method can include mainstem bronchi into the lung regions because of partial volume effect, as shown in Fig. 1(c). This may not be consistent across slices and therefore causes the lung boundary to have an irregular appearance, especially in coronal and sagittal views [4. To remove indentations and salience caused by gray-level based method and obtain consistent appearance across slices, smoothing the lung boundary is required after segmentation.

\footnotetext{
^ Corresponding author, Seoul Women's University, Phone: 82-2-970-5756.
} 


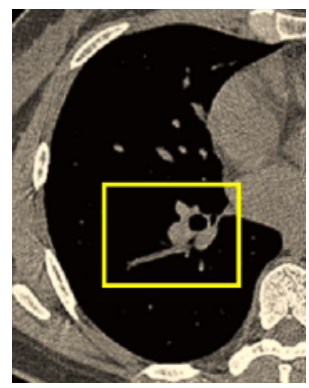

(a)

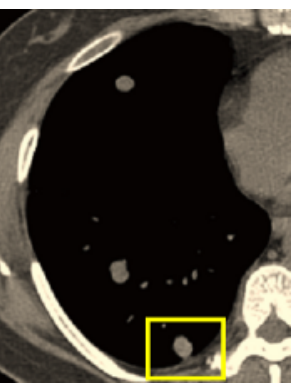

(b)

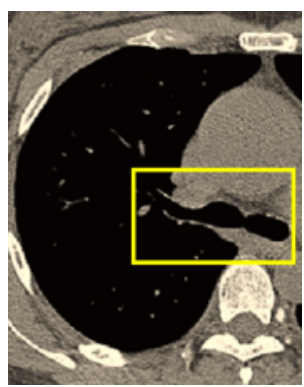

(c)

Fig. 1. Original axial CT slices in which gray-level based segmentation method gives rise to indentations and salience of lung regions: (a) axial CT slice with pulmonary vessels, (b) axial CT slice with a pleural nodule, (c) axial CT slice with mainstem bronchi

Several approaches have been suggested for smoothing lung boundary in chest CT images. To compensate the lost pleural nodules, Kanazawa [5] and Ko 6] utilized curvature of points on the initial lung contour. Curvature-based method replaces the contour with rapidly changing curvature using a straight line. To calculate the curvature, however, time-consuming contour tracking is required. Armato [2] rectified indentations that corresponds to pleural nodules by rolling a ball along the lung contour. If the ball overlaps the contour at two contour pixels, linear interpolation is used to identify new contour pixels. However, balls of different radius have to be applied in succession to remove concavities of different dimensions. Shen [7] proposed an algorithm for surface smoothing within a volume of interest (VOI). To segment the pleural lung nodules from the VOI, position and orientation of each point on lung surfaces are transformed into intensity value of $2 \mathrm{D}$ image, and then high intensity region which corresponds to nodule is eliminated from this image. This algorithm requires user manipulation for VOI selection and is suitable only for local surface smoothing. For 3D smoothing of the lung surfaces near mediastinum, Ukil [4] performed 3D morphological closing with an ellipsoidal mask using information from the segmented airway tree. Even though this method gives well-smoothed lung surfaces, it requires too excessive time about 5-6 minutes for 3D smoothing of datasets with 300-600 image slices.

To deal with the above limitations, we propose a new method for smoothing segmented lung boundary in chest CT images through scan line search. To track the contour points and find rapidly changing curvature efficiently, we search for intersection points between scan lines and lung contour. To reduce the number of high curvature point, we apply $2 \mathrm{D}$ closing in axial slice, as a pre-processing step. For consistent appearance between lung contours in neighboring axial slices, we apply $2 \mathrm{D}$ closing in coronal slice. In order not to distort the lung contour away from mediastinum, this operation is performed within predefined subvolume near mediastinum. 


\section{Methods}

The proposed method for smoothing lung boundary is outlined in Fig. 2, Our method consists of three main steps: lung segmentation, smoothing in axial slice and subvolume smoothing in coronal slice.

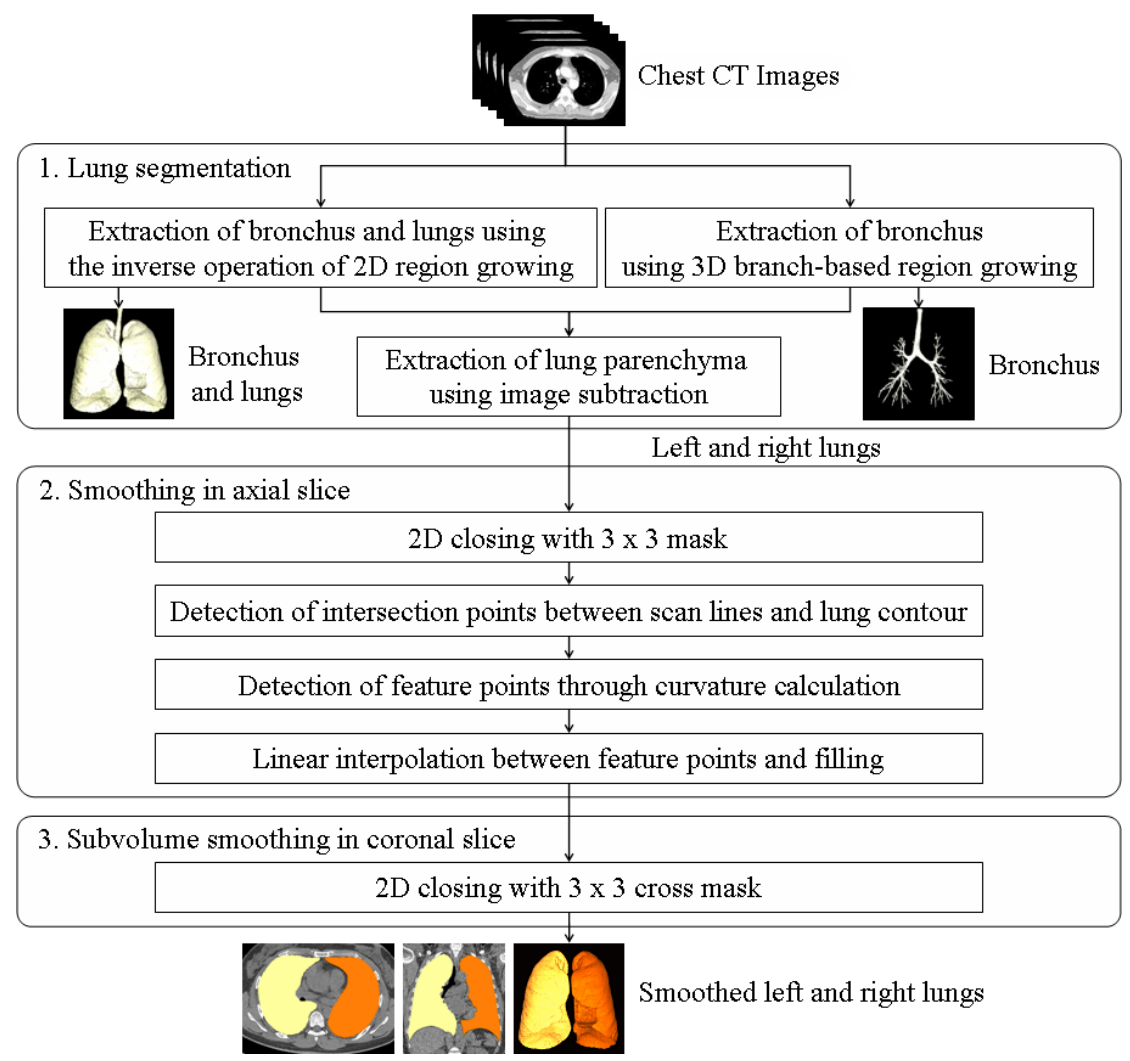

Fig. 2. The pipeline of proposed method for smoothing segmented lung boundary in chest CT images

\subsection{Lung Segmentation}

The lung boundary is extracted by our automatic segmentation method 3, which is based on density and morphology information in chest CT images. To extract left and right lungs, we use the inverse operation of $2 \mathrm{D}$ region growing (2D iRG) that segments the thorax from the background and then the lungs from the thorax. Since the density of bronchus is similar to that of the lungs in CT images, the lungs segmented by 2D iRG still contain the bronchus.

To delineate the bronchus from the lungs, we first extract bronchus and then subtract the result from the segmentation result of bronchus and lungs in 
voxel-by-voxel manner. To segment bronchus without leakage into lung parenchyma, we use our 3D branch-based region growing [8] that changes the growing condition according to morphology of bronchus. This method differentiates trachea from left and right bronchi based on branch bifurcation and applies different threshold values. For trachea, 3D region growing uses the threshold value determined by optimal thresholding [9]. Fig. 3] shows the process of region growing. Region grows from seed point until bifurcation is found. To detect bifurcation, we perform 3D connected component labeling with 26-connectivity to the grown region in every step and adopt the location where the number of connected component becomes two. For left and right mainstem bronchi, we calculate a new threshold value $\mu-h \sigma$ where $\mu$ and $\sigma$ is respectively mean and standard deviation of gray-level values of voxels that corresponds to trachea and $h$ is a parameter that controls the ease of region growing. To increase robustness of bronchus extraction, we apply additional processing such as median filtering and morphological operations.

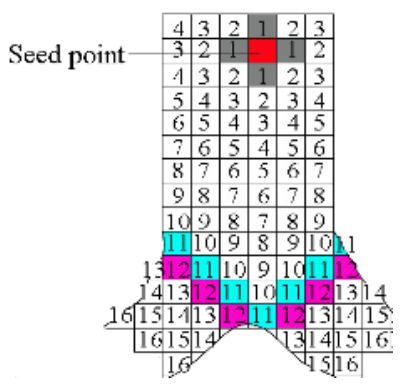

Fig. 3. 3D branch-based region growing. 3D region growing with 6-connectivity is simplified to the $2 \mathrm{D}$ region growing with 4 -connectivity. The number written in each voxel describes growing step.

\subsection{Smoothing in Axial Slice}

Since the lung segmentation entirely removes trachea and mainstem bronchi, inconsistent extraction of bronchus across slices can be solved. However, concavities from pleural nodules and pulmonary vessels are still remained. For smoothing the segmented lung contour, curvature-based method is commonly used [56]. This method assumes that a rapid change in curvature at points on the lung contour indicates a nodule, large vessel or bronchus, and corrects the lung contour by inserting a border segment. To calculate the curvature of a point, we have to know the previous and next point on lung contour, and therefore contour tracking is required, which takes excessive processing time. To smooth segmented lung contour based on curvature without this time-consuming process, we propose smoothing in axial slice through scan line search.

Smoothing in axial slice tracks the points on lung contour using scan line search, and fills out unwanted cavities by connecting high curvature points. For 
each lung, we first search for intersection points between scan lines and lung contour by scanning each 2D axial CT slice in horizontal direction at fixed intervals, as shown in Fig. 4(a). For contour tracking, we classify intersection points of $i^{\text {th }}$ scan line into three groups: first point $I_{i}^{\text {first }}(x, y)$, last point $I_{i}^{\text {last }}(x, y)$ and middle points $I_{i}^{\text {middle }_{j}}(x, y)$. By tracking points of identical group on neighboring scan lines, as shown in Fig. 4(b), we can calculate the curvature of point on a scan line. As the scan line interval becomes shorter, we can get more accurate curvature. The scan line interval is experimentally set to 4 pixels.

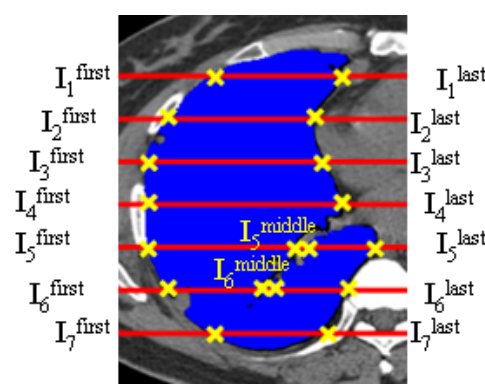

(a)

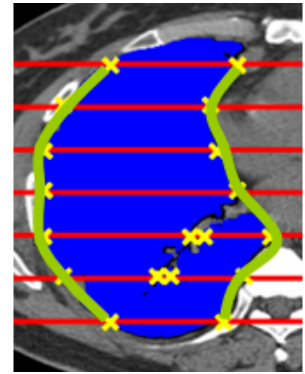

(b)

Fig. 4. Scan line search: (a) detection of intersection points between scan lines and lung contour, (b) tracking of lung contour for curvature calculation

Based on this approximation, we calculate the curvature of the first and last intersection point in each scan line using Eq. (1).

$$
\kappa_{i}=\frac{\left(x_{i-1}-x_{i}\right) \times\left(y_{i-1}-2 y_{i}+y_{i+1}\right)-\left(y_{i-1}-y_{i}\right) \times\left(x_{i-1}-2 x_{i}+x_{i+1}\right)}{\left[\left(x_{i-1}-x_{i}\right)^{2}+\left(y_{i-1}-y_{i}\right)^{2}\right]^{3 / 2}}
$$

where $\kappa_{i}$ is the curvature and $\left(x_{i}, y_{i}\right)$ is the coordinates of $I_{i}(x, y)$. To calculate the curvature of the first intersection point of $i^{\text {th }}$ scan line, $I_{i-1}^{\text {first }}(x, y)$ and $I_{i+1}^{f i r s t}(x, y)$ is required as well as $I_{i}^{f i r s t}(x, y)$. After calculating the curvature of intersection points, we adopt high curvature points as feature points. We determine the curvature is high when its absolute value is larger than 0.2. All middle intersection points are also added to feature points to improve accuracy of the above approximation.

The number of feature points detected from left and right lungs is 41 per axial slice, on average. To reduce the number of feature points, we apply $2 \mathrm{D}$ closing operation with $3 \times 3$ mask to each slice, as a pre-processing step. In order to distort lung contour as little as possible, the iteration number is experimentally set to 2 . As a result, many concave indentations near mediastinum become gentle and the number of feature points per slice is reduced to 35 . 
After finding feature points through curvature calculation, we connect all different feature points by linear interpolation when the distance between two points is in the pre-defined range. Experimentally, this range is set from 3 to 40 pixels. The regions that are encompassed by connecting lines are filled by $2 \mathrm{D}$ region growing.

\subsection{Subvolume Smoothing in Coronal Slice}

In smoothing in axial slice, detected feature points and smoothed regions are different across slices. For this reason, smoothed lung contour in one slice can be inconsistent with that of neighboring slice. In coronal or sagittal view, rugged boundary still remains. To solve this problem, we apply $2 \mathrm{D}$ closing operation in coronal slice with $3 \times 3$ cross mask.

Closing consists of dilation followed by erosion. For 2D dilation operation with $3 \times 3$ cross mask, we perform $2 \mathrm{D}$ convolution with $3 \times 3$ cross mask and maximum filtering. For $2 \mathrm{D}$ erosion operation, $2 \mathrm{D}$ convolution is followed by minimum filtering. Fig. 5 shows the process of $2 \mathrm{D}$ dilation operation with 3 $\times 3$ cross mask. We first convolve the input image with cross mask, and apply gray-level thresholding to convolved image with threshold 30, as shown in Fig. 5)(a) and (b). Maximum filtering with $3 \times 3$ mask sets each pixel in Fig. 5)(c) as the maximum value among all the pixel values of thresholded image in the neighborhood of size $3 \times 3$.

2 D closing operation with $3 \times 3$ cross mask can lead to smooth lung contour in coronal slice while applying the operation with $3 \times 3$ mask iteratively can cause jagged lung contour. To avoid unwanted distortion of lung contour, closing

\begin{tabular}{|l|c|c|c|c|c|}
\hline 0 & 0 & 0 & 0 & 0 & 0 \\
\hline 0 & 0 & 0 & 0 & 0 & 0 \\
\hline 0 & 0 & 10 & 10 & 10 & 0 \\
\hline 0 & 0 & 10 & 10 & 0 & 0 \\
\hline 0 & 0 & 0 & 0 & 0 & 0 \\
\hline 0 & 0 & 0 & 0 & 0 & 0 \\
\hline
\end{tabular}$\otimes$\begin{tabular}{|l|l|l}
\hline 0 & 1 & 0 \\
\hline 1 & 1 & 1 \\
\hline 0 & 1 & 0 \\
\hline
\end{tabular}$=$\begin{tabular}{c|c|c|c|c|c|c|}
\hline 0 & 0 & 0 & 0 & 0 & 0 \\
\hline 0 & 0 & 10 & 10 & 10 & 0 \\
\hline 0 & 10 & 30 & 40 & 20 & 10 \\
\hline 0 & 10 & 30 & 30 & 20 & 0 \\
\hline 0 & 0 & 10 & 10 & 0 & 0 \\
\hline 0 & 0 & 0 & 0 & 0 & 0 \\
\hline
\end{tabular}

\begin{tabular}{|l|l|l|l|l|l|}
\hline 0 & 0 & 0 & 0 & 0 & 0 \\
\hline 0 & 0 & 0 & 0 & 0 & 0 \\
\hline 0 & 0 & 10 & 10 & 0 & 0 \\
\hline 0 & 0 & 10 & 10 & 0 & 0 \\
\hline 0 & 0 & 0 & 0 & 0 & 0 \\
\hline 0 & 0 & 0 & 0 & 0 & 0 \\
\hline
\end{tabular}

(b) (a)

\begin{tabular}{|l|c|c|c|c|c|}
\hline 0 & 0 & 0 & 0 & 0 & 0 \\
\hline 0 & 10 & 10 & 10 & 10 & 0 \\
\hline 0 & 10 & 10 & 10 & 10 & 0 \\
\hline 0 & 10 & 10 & 10 & 10 & 0 \\
\hline 0 & 10 & 10 & 10 & 10 & 0 \\
\hline 0 & 0 & 0 & 0 & 0 & 0 \\
\hline
\end{tabular}

(c)

Fig. 5. 2D dilation with $3 \times 3$ cross mask: (a) convolution, (b) the result image after applying gray-level thresholding with threshold value $30,(\mathrm{c})$ the result image after applying maximum filter 
operation is applied only within pre-defined subvolume near mediastinum. Fig. 6(a) shows the subvolume, which is in the range of $0.4 \times$ width $<x<0.7 \times$ width, $0.4 \times$ height $<\mathrm{y}<0.7 \times$ height, $0.4 \times$ depth $<\mathrm{z}<0.8 \times$ depth. Fig. 6(b) and (c) shows the effect of subvolume smoothing in coronal slice. After subvolume smoothing in coronal slice, inconsistency across axial slices is much reduced.

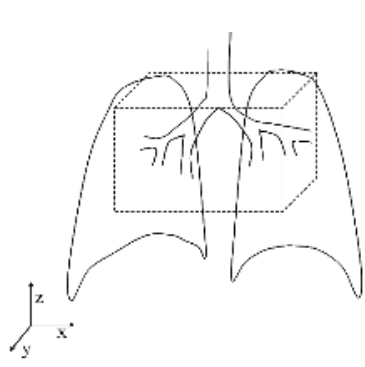

(a)

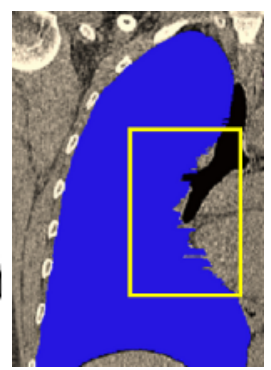

(b)

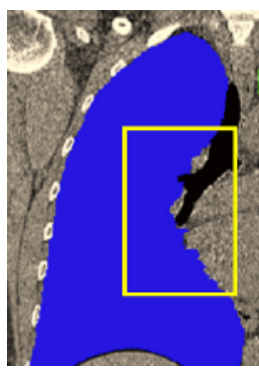

(c)

Fig. 6. Subvolume smoothing in coronal slice: (a) the range of subvolume, (b) the result before subvolume smoothing in coronal slice, (c) the result after subvolume smoothing in coronal slice

\section{Experimental Results}

All our implementation and test were performed on an Intel Pentium 4 PC containing 2.5 GHz CPU and 2.0 GB of main memory. Our method has been applied to six patients with pulmonary nodule of 16-channel chest CT scans whose properties are described in Table 1. The CT images were obtained with LightSpeed Ultra (GE Healthcare) and Sensation16 (Siemens) multi-detector row CT scanner. Experimental dataset contains 222-446 slices with a slice thickness of $0.75-1.25 \mathrm{~mm}$ and a reconstruction size of $512 \times 512$ pixels.

Fig. 7 shows the lung contours smoothed by proposed method in axial and coronal CT slices. In Fig. 7(b) and (e), lung nodules and pulmonary vessels are excluded from the lung contours. The result in Fig. 7(c) and (f) shows that our smoothing algorithm compensates for these lost parts.

Table 1. Image conditions of experimental datasets

\begin{tabular}{cccc}
\hline Subject & Slice number & Pixel size $(\mathrm{mm})$ & Slice thickness $(\mathrm{mm})$ \\
\hline 1 & 222 & $0.63 \times 0.63$ & 1.25 \\
2 & 291 & $0.66 \times 0.66$ & 1.0 \\
3 & 279 & $0.64 \times 0.64$ & 1.25 \\
4 & 258 & $0.59 \times 0.59$ & 1.25 \\
5 & 446 & $0.64 \times 0.64$ & 0.75 \\
6 & 305 & $0.59 \times 0.59$ & 2.0 \\
\hline
\end{tabular}




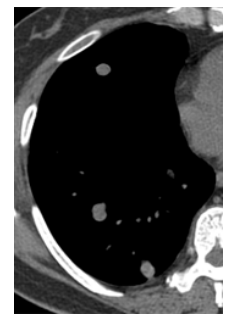

(a)

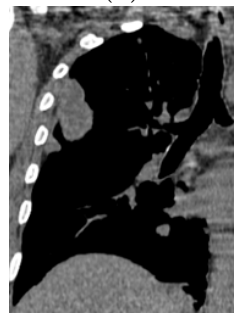

(d)

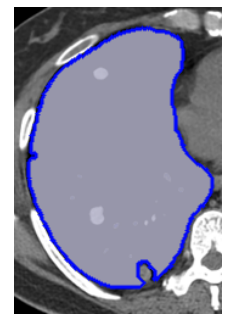

(b)

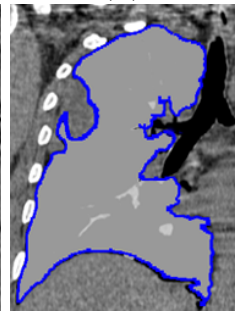

(e)

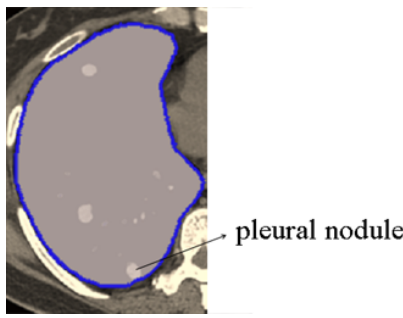

(c)

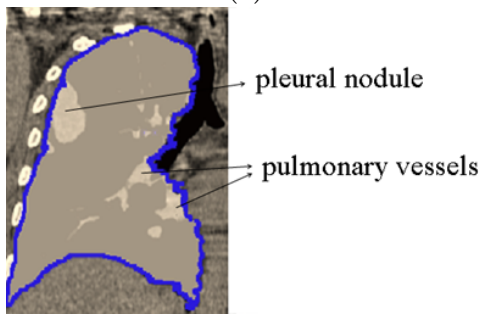

(f)

Fig. 7. The result of smoothing: (a) original axial CT slice, (b) automatic segmentation of (a) by [3, (c) smoothing of (b) by proposed method, (d) original coronal CT slice, (e) automatic segmentation of (d) by [3, (f) smoothing of (e) by proposed method

Fig. 8 shows the 3D rendered view of the segmentation result after smoothing. Figures show that the result has smooth lung boundary in all aspects and consistent appearance across slices.

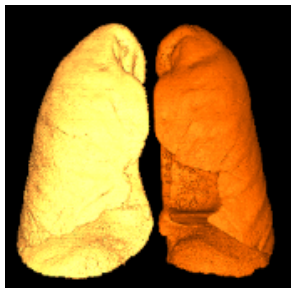

(a)

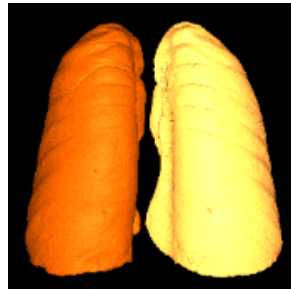

(b)

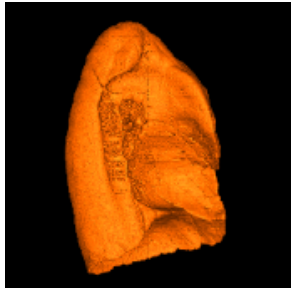

(c)

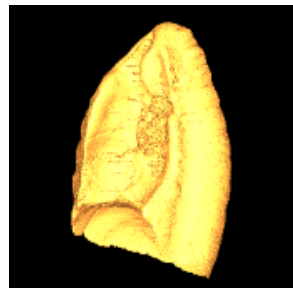

(d)

Fig. 8. 3D rendered view of segmentation result after smoothing: (a) anterior view, (b) posterior view, (c) medial view of left lung, (d) medial view of right lung

To evaluate the accuracy of segmented lung contour, we calculated the average of absolute value of curvature over mediastinal axial slices before and after smoothing. For this evaluation, we tracked the contour using chain code [10], which first picks a starting pixel and finds the next pixel on lung contour by searching the eight directions surrounding the current pixel. The value calculated before and after smoothing was 0.0496 and 0.0371 , respectively. This 
result shows that the smoothness of lung contour considerably increased after smoothing.

Processing time for smoothing segmented lung boundary was measured from 6 patient datasets. Total processing time was 5.62 seconds on average, as shown in Fig. 9. For smoothing in axial slice and subvolume smoothing in coronal slice, 3.62 and 2.0 seconds were required, respectively. To prove efficiency of proposed scan line search, we compared the contour tracking time between our method and conventional contour tracking technique. For conventional method, we implemented contour tracking using chain code. From 6 patient datasets, the average contour tracking time of conventional method was 1.281 seconds. On the other hand, the average time needed for scan line search was 0.286 seconds.

\section{D smoothing in axial slice $\square$ 2D subvolume smoothing in coronal slice}

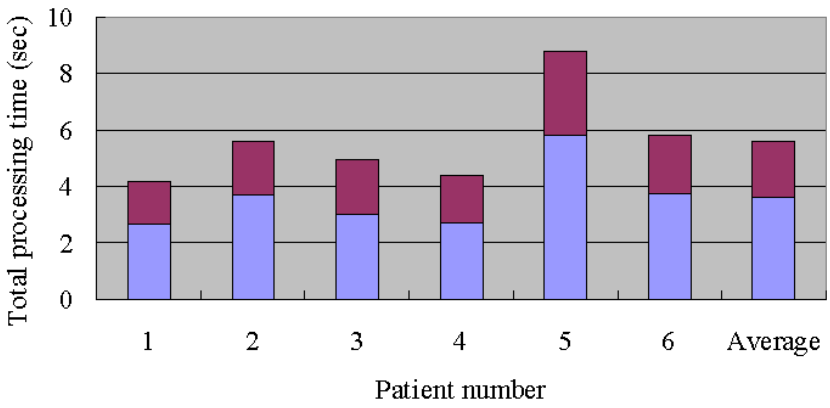

Fig. 9. Total processing time of proposed method for smoothing segmented lung boundary

\section{Conclusion}

We have developed a new method for smoothing segmented lung boundary in chest CT images. Using scan line search and curvature calculation, we can smooth lung boundary identified by our automatic segmentation method. Scan line search allows us to track the points and find rapidly changing curvature on lung contour efficiently. 2D closing in axial slice can reduce the number of rapidly changing curvature points. Subvolume smoothing in coronal slice provides consistent appearance between lung contours in neighboring axial slices. Six patient datasets with lung nodules have been used for the performance evaluation with the aspects of visual inspection, accuracy and processing time. The results of our method show that the smoothness of lung contour considerably increased by compensating for pulmonary vessels near mediastinum and pleural nodules. Proposed method have been successfully used for lung nodule matching [1112] and can be used for quantitative evaluation such as measurement of nodule growth rate. 


\section{Acknowledgements}

This study is supported by research fund from Seoul Womens University, 2006.

\section{References}

1. Hu, S., Hoffman, E.A., Reinhardt, J.M.: Accurate Lung Segmentation for Accurate Quantitation of Volumetric X-Ray CT Images. IEEE Transactions on Medical Imaging, Vol. 20, No. 6 (2001) 490-498

2. Armato, S.G., Sensakovic, W.F.: Automated Lung Segmentation for Thoracic CT: Impact on Computer-Aided Diagnosis. Academic Radiology, Vol.11 (2004) 10111021

3. Yim, Y., Hong, H.: Automatic Segmentation of Pulmonary Structures in Chest CT Images. Lecture Notes in Computer Science, Vol. 3773 (2005) 654-662

4. Ukil, S., Reinhardt, J.M.: Smoothing Lung Segmentation Surfaces in 3D X-ray CT Images using Anatomic Guidance. Proceeding of SPIE Conference on Medical Imaging, Vol. 5340 (2004) 1066-1075

5. Kanazawa, K., Kawata, Y., Niki, N., et al.: Computer-aided diagnosis for pulmonary nodules based on helical CT images. Computerized Medical Imaging and Graphics, Vol. 22 (1998) 157-167

6. Ko, J.P., Betke, M., Chest CT: Automated Nodule Detection and Assessment of Change over Time - Preliminary Experience. Radiology, Vol. 218 (2001) 267-273

7. Shen, H., Goebel, B., Odry, B.: A New Algorithm for Local Surface Smoothing with Application to Chest Wall Nodule Segmentation in Lung CT Data. SPIE Conference on Medical Imaging, Vol. 5370 (2004) 1519-1526

8. Yim, Y., Hong, H.: Automatic Segmentation of Pulmonary Structures in Chest CT Images. accepted at Computer Assisted Radiology and Surgery $20^{t h}$ International Congress and Exhibition (2006)

9. Gonzalez, R.C., Woods, R.E.: Digital Image Processing. Addison-Wesley Publishing Company (1992)

10. Baxes, G.A.: Digital Image Processing: Principles and Applications. John Wiley \& Sons, New York (1994)

11. Hong, H., Lee, J.J., Yim, Y., Shin, Y.G.: Automatic Segmentation and Registration of lung surfaces in temporal chest CT scans. Lecture Notes in Computer Science, Vol. 3523 (2005) 463-470

12. Hong, H., Lee, J.J., Yim, Y., Shin, Y.G.: Automatic lung nodule matching for the follow-up in temporal computed tomography scans. Proc. of RSNA 2005, Chicago, USA (2005) 